\title{
Ictericia secundaria a cistoadenoma seroso pancreático gigante
}

\section{Jaundice secondary to giant pancreatic serous cystoadenoma}

\author{
S. Goñi ${ }^{1}$, J.J. Vila ${ }^{1}$, J. Valle ${ }^{2}$, J. Martín Cuartero ${ }^{3}$, D. Ruiz-Clavijo ${ }^{1}$, F.J. Jiménez Pérez ${ }^{1}$
}

\section{RESUMEN}

El cistoadenoma seroso es el segundo tumor quístico más frecuente del páncreas y representa el 1-2\% de todas las neoplasias exocrinas pancreáticas. Recientemente, gracias a las mejoras en las técnicas de imagen, la identificación de las lesiones quísticas pancreáticas es cada vez más frecuente. El diagnóstico diferencial ha de hacerse con el cistoadenoma mucinoso, debido al potencial maligno de esta última entidad. En esta nota clínica describimos el caso de una paciente con ictericia indolora y colestasis, con diagnóstico final de cistoadenoma seroso pancreático.

Palabras clave. Cistoadenoma seroso. Páncreas. Colestasis. Neoplasias pancreáticas.

\begin{abstract}
Serous cystadenoma is the second most frequent pancreatic cystic neoplasm and accounts for $1-2 \%$ of exocrine neoplasms of the pancreas. Recently, they have been identified more frequently, due to the improvement in imaging techniques. Differential diagnosis should be performed with mucinous cystoadenoma, due to the latter's potential for malignant transformation. We present the case of a female patient who underwent examination for painless jaundice and cholestasis, with a final diagnosis of pancreatic serous cystoadenoma.
\end{abstract}

Key words. Serous cystadenoma. Pancreas. Cholestasis. Pancreatic neoplasms.
1. Servicio de Digestivo. Hospital de Navarra. Pamplona.

2. Servicio de Medicina Interna. Hospital Reina Sofía. Tudela.

3. Servicio de Radiología. Hospital Reina Sofía. Tudela.

Recepción el 29 de enero de 2010

Aceptación provisional el 15 de abril de 2010

Aceptación definitiva el 30 de abril de 2010

\section{Correspondencia}

Silvia Goñi Esarte

Servicio de Digestivo

Hospital de Navarra

Irunlarrea, 3

31008 Pamplona

E-mail: silvigoni@hotmail.com 


\section{INTRODUCCIÓN}

Los cistoadenomas serosos son tumores benignos del páncreas, que también se conocen como adenomas microquísticos o cistoadenomas ricos en glucógeno ${ }^{1}$. Dos tercios de los pacientes presentan manifestaciones clínicas como dolor abdominal, náuseas, vómitos, masa abdominal o pérdida de peso. El tercio restante se descubren accidentalmente en una exploración física de rutina o autopsias ${ }^{2}$. Consideramos relevante la presentación de este caso puesto que el diagnóstico diferencial es fundamental, dado que las formas serosas pueden ser manejadas de forma segura mediante la observación, mientras que las formas mucinosas deben ser resecadas debido a su alto potencial maligno ${ }^{3}$.

\section{CASO CLÍNICO}

Mujer de 89 años de edad, con antecedentes de hipertensión arterial, diabetes mellitus tipo 2, fibrilación aurícular y EPOC, que ingresa por ictericia cutaneomucosa de 15 días de evolución, acompañada de prurito intenso, coluria y astenia. No refería anorexia ni dolor abdominal. En la exploración física se palpaba zona indurada no dolorosa en epigastrio siendo el resto de la exploración irrelevante.

En la analítica de sangre destacaban los siguientes parámetros: bilirrubina total de 12,88 $\mathrm{mg} / \mathrm{dl}$ (rango, 0,1-1), bilirrubina directa de 9,92 $\mathrm{mg} / \mathrm{dl}$ (rango, 0,01-0,25), AST de $244 \mathrm{U} / \mathrm{L}$ (rango, 5-35), ALT de $272 \mathrm{U} / \mathrm{L}$ (rango, 5-44), GGT de 420 $\mathrm{U} / \mathrm{L}$ (rango, 5-46) y fosfatasa alcalina de $1.926 \mathrm{U} / \mathrm{L}$ (rango, 90-360) con resto de parámetros bioquímicos, incluidos amilasa y lipasa, dentro de la normalidad.

Se realizó una ecografía de urgencia, en la cual se puso de manifiesto una masa voluminosa localizada en la cabeza pancreática, de predominio quístico multitabicada y de contornos lobulados, con dilatación de la vía biliar principal y las vías biliares intrahepáticas. Se practicó una tomografía computarizada, en la que se objetivó una masa en la cabeza del páncreas, de aspecto multiquístico con tabiques y calcificaciones «en puesta de sol» (Fig. 1), que provocaba dilatación de la vía biliar y de Wirsung; compatible con cistoadenoma seroso pancreático. Además presentaba una vesícula biliar muy distendida (Fig. 2) y marcada dilatación de la vía biliar intrahepática y del colédoco (Fig. 3).

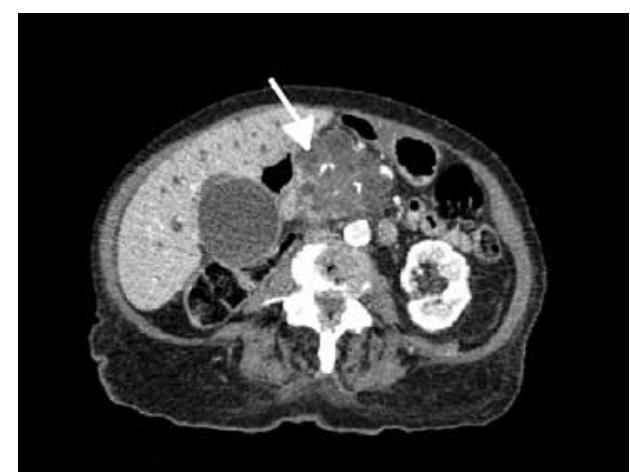

Figura 1. Hallazgos en la tomografía computarizada (corte axial). Masa en cabeza de páncreas de unos $6 \times 10 \mathrm{~cm}$, tabicada y con calcificaciones «en puesta de sol» (flecha).

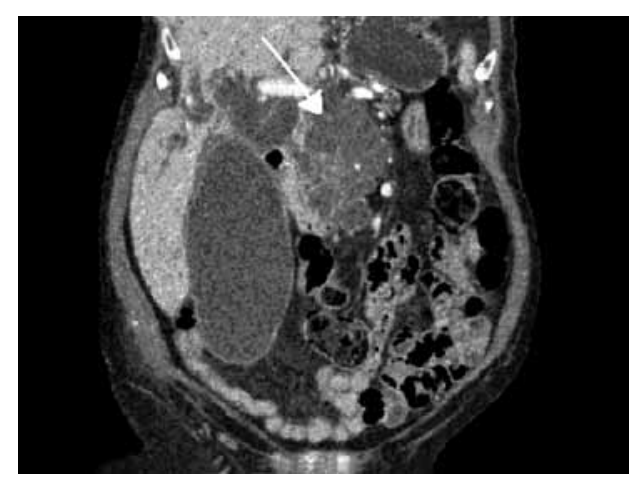

Figura 2. Hallazgos en la tomografía computarizada (corte coronal). Se aprecia la vesícula biliar muy distendida de $18 \mathrm{~cm}$ de longitud y $7 \mathrm{~cm}$ de diámetro y la masa pancreática (flecha).

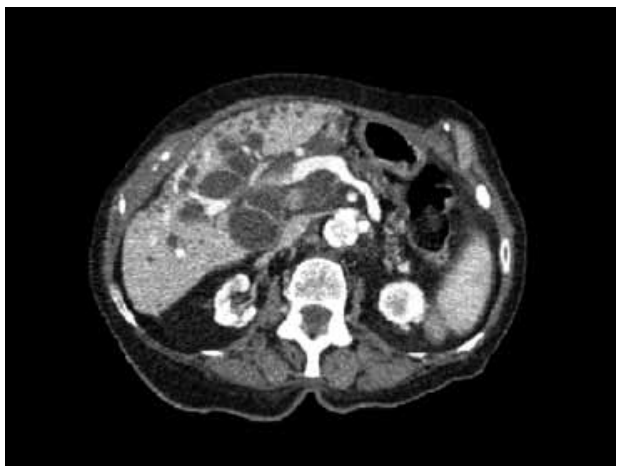

Figura 3. Imagen de tomografía computarizada en la que se observa marcada dilatación de la vía biliar intrahepática. 
Dada la edad y los antecedentes personales de la paciente no fue considerada subsidiaria de intervención quirúrgica y se practicó una colangiopancreatografía retrógrada endoscópica (CPRE). Al realizar la CPRE se objetivó cierta dificultad de paso del endoscopio a través de rodilla duodenal superior, en probable relación con compresión por la lesión primaria pancreática. En la colangiografía se apreció una gran dilatación de colédoco proximal con estenosis distal de aproximadamente $3-4 \mathrm{~cm}$ de longitud. Se colocó una prótesis biliar metálica autoexpandible tipo Wallstent (Boston Scientific, Natick, MA, USA) de $8 \mathrm{~cm}$ de longitud y $1 \mathrm{~cm}$ de calibre $\mathrm{sin}$ incidencias, comprobando la correcta colocación y drenaje posterior. La paciente presentó una progresiva despigmentación cutaneomucosa con descenso de niveles de bilirrubina. Finalmente fue dada de alta asintomática.

\section{DISCUSIÓN}

Los tumores quísticos del páncreas son relativamente infrecuentes, constituyendo el 5\% de todas las neoplasias pancreáticas ${ }^{4}$. Los cistoadenomas serosos son casi siempre benignos y representan el $15 \%$ de todos los tumores quísticos del páncreas $^{5}$. Afectan sobre todo a mujeres (80\%) en la séptima u octava década de la vida, localizándose la mayoría en el cuerpo o la cola ${ }^{6}$. Pueden ocurrir de forma esporádica o asociados con la enfermedad de von Hippel-Lindau? ${ }^{7}$ La mayor parte de los pacientes debutan con dolor o molestias digestivas inespecíficas, pudiendo debutar como masa palpable si alcanza gran tamaño. Cada vez se detectan más tumores asintomáticos de forma incidental durante la valoración de otros procesos por técnicas de imagen.

El diagnóstico de los cistoadenomas serosos se basa en técnicas de imagen como la tomografía computarizada (TC), la ecografía y la ecoendoscopia (EE). La imagen patognomónica en la TC es una masa esponjosa con calcificación central "en puesta de sol», hallazgo que sólo aparece en aproximadamente el $10 \%$ de los pacientes $^{8}$. Los síntomas, la precisión del diagnóstico preoperatorio, la seguridad de la resección y los riesgos del manejo conservador, juegan un papel importante en la decisión del tratamiento en los cistoadenomas serosos.

Dado el potencial maligno de estas lesiones inferior al $1 \%^{9}$ y la mortalidad asociada con la cirugía pancreática, algunos autores recomiendan un manejo conservador en pacientes asintomáticos sin obstrucción biliar, pacientes de edad avanzada o con elevado riesgo quirúrgico ${ }^{10,11}$. Esta actitud tiene el riesgo de crecimiento progresivo del tumor y el desarrollo de complicaciones como la hemorragia, la ictericia obstructiva, la insuficiencia pancreática o la obstrucción del tracto gastrointestinal.

La mayoría de los pacientes sintomáticos son manejados mediante la resección quirúrgica, que puede consistir en una intervención de Whipple o una pancreatectomía distal, según la localización anatómi$\mathrm{ca}^{12}$.

En nuestro caso, dada la edad de la paciente y comorbilidades, se decidió realizar un tratamiento paliativo de los síntomas con la colocación de una prótesis metálica en la vía biliar.

En conclusión, describimos un caso de rara presentación de obstrucción biliar por cistoadenoma seroso gigante de cabeza de páncreas, en el que por comorbilidades de la paciente se decidió tratamiento no quirúrgico, con buena evolución.

\section{BIBLIOGRAFÍA}

1. Omeroglu A, Paner GP, Ciesla mC, Hartman G. Serous mycrocistic adenomas of the pancreas. Arch Pathol Lab Med 2001; 125: 16131614.

2. Vernadakis S, Kaiser GM, Christodoulou E, Mathe Z, Troullinakis M, Bankfalvi A et al. Enormous serous mycrocistic adenoma of the pancreas. JOP 2009; 10: 332-334.

3. Fernández JA, Sánchez F, Cáscales PA, Robles R, Frutos MD, MARÍn C et al. Cistoadenoma seroso unilocular macroquístico de páncreas. Cir Esp 2006; 79: 57-60.

4. Malur PR, Suranagi VV, Bannur HB, Kulgod S. Serous microcystic adenoma of the pancreas. Indian J Pathol Microbiol 2009; 52: 408-410. 
5. Fernández-del Castillo C, Warshaw Al. Cystic tumors of the pancreas. Surg Clin North Am 1995; 75: 1001-1016.

6. Slukin II, Hafez GR, Niederhuber JE, Warner TF. Combined serous microcystic adenoma and well differentiated endocrine pancreatic neoplasm: a case report and review of literature. Arch Pathol Lab Med 2003; 127: 1369-1372.

7. Mohr VH, Vormeyer AO, Zhuang Z, Libutti SK, Walther MM, Choyke PL et al. Histopathology and molecular genetics of multiple cysts and microcystic (serous) adenomas of the pancreas in von Hippel-Lindau patients. Am J Pathol 2000; 157: 1615-1621.

8. Warshaw AL, Compton CC, Lewandrowski K, CarDenosa G, Mueller PR. Cystic tumors of the pancreas. New clinical, radiologic and pathologic observations in 67 patients. Ann Surg 1990; 212: 432-443.
9. Abe H, Kubota K, Mori M, Miki K, Minangawa M, NoIE $T$ et al. Serous cystadenoma of the pancreas with invasive growth: benign or malignant? Am J Gastroenterol 1998; 93: 1963-1966.

10. Tampi C, Mullerpatan P, Shah R, Jagannath P, Zimmermann A. Microcystic serous cystoadenoma of the pancreas: a report of two cases with one of diffuse presentation. Pancreatology 2006; 6: 248-253.

11. Galanis C, Zamani A, Cameron JL, Campbell Ka, Lillemoe KD, Caparrelli D et al. Resected serous cystic neoplasms of the pancreas: a review of 158 patients with recommendations for treatment. J Gastrointest Surg 2007; 11: 820-826.

12. Hashimoto M, Watanabe G, Matsuda M, Mori M. Serous cystic neoplasm of the pancreas-indications for surgery. Hepatogastroenterology 2006; 53: 950-952. 